\title{
Validity of Force Concept Inventory evaluated by students' explanations and confirmation using modified item response curve
}

\author{
Yoshihiko Shoji* \\ Laboratory of Advanced Science and Technology for Industry, University of Hyogo, \\ NweSUBARU/SPring-8, Kamigori-cho, Ako-gun, Hyogo, Japan 678-1205 \\ Shuji Munejiriఠ \\ Graduate School of Advanced Science and Engineering, Hiroshima University, \\ 1-7-1 Kagamiyama, Higashi-Hiroshima City Hiroshima, Japan 739-8521 \\ Eiko Kaga \\ Graduate School of Integrated Arts and Sciences, Hiroshima University, \\ 1-7-1 Kagamiyama, Higashi-Hiroshima City Hiroshima, Japan 739-8521
}

(Received 18 February 2021; accepted 23 August 2021; published 20 September 2021)

\begin{abstract}
In this study, we evaluate the validity of question 29 (Q.29) in the Force Concept Inventory (FCI), which requires an understanding of atmospheric pressure. At least in some Japanese physics classes, the rate of the correct answer to Q.29 is reduced between the pretest and post-test. To examine this deterioration of a student's response, we analyzed 429 explanations written by 358 respondents about the force described in the question. Many learners, who were not aware of atmospheric force, chose the correct answer. On the other hand, many of the wrong respondents had learned about air pressure but did not understand it. As a result, postinstruction learners tended to have a worse rate of correct response. This made Q.29 less sensitive to a Japanese student's ability. This characteristic feature of Q.29 is visually confirmed by a modified item response curve, which represents correlation between the response to one item and the total score of the other items.
\end{abstract}

DOI: 10.1103/PhysRevPhysEducRes.17.020120

\section{INTRODUCTION}

The Force Concept Inventory (FCI) is a widely used test in physics education [1,2]. It is a 30 -item, five-choice survey to probe conceptual understanding of Newtonian mechanics. The FCI is most often used as an assessment tool to measure the effectiveness of classroom instruction. This application requires a high-test reliability, which means that a class should provide the same responses under the same conditions. The FCI is also used as a measure of understanding of Newtonian mechanics. This application requires a high validity, which ideally means one-to-one correspondence between the FCI score and a student's ability. Although many studies have proven that the reliability and validity of the FCI are excellent, many researchers are still studying these characteristics of the FCI because of its importance.

\footnotetext{
*shoji@lasti.u-hyogo.ac.jp
}

Published by the American Physical Society under the terms of the Creative Commons Attribution 4.0 International license. Further distribution of this work must maintain attribution to the author(s) and the published article's title, journal citation, and DOI.
When the FCI is used as an assessment of educational effectiveness, the test is administered at the beginning of a class (pre-instruction-test; pretest) and at the end of the same class (postinstruction-test; post-test). An increase in correct answers is recognized as a good measure of educational effectiveness. Commonly, Hake's normalized gain [3] or effective size (Cohen's $d$ ) [4] is used. In this process, some instructors received a reduction of the rate of correct responses to a special question, which could not be explained by random fluctuation. Both systematic gain, a change from an incorrect (or negative) response to a correct (or positive) response, and systematic loss, a change from a correct response to an incorrect response, should be explained by students' understanding and their thought processes. Here, "systematic loss" means a loss not caused by a statistical fluctuation. Of the 30 items on the FCI, one item (Q.15) is known to have systematic loss. Wilson and Low clearly explained the thought process using the conflict between Newton's second law and third law accompanied by the concept of net force $[5,6]$. The subject of this article is another item (Q.29) showing a systematic loss, due to a different thought process than that of Q.15.

A systematic loss for Q.29 would not be a rare event, at least not in Japan. A Japan-wide survey directed by Lang 
suggested its emergence [7] and in fact, we observed a systematic loss in three classes at the University of Hyogo and a high school. We analyzed the explanations by respondents on the force suggested in the question. We should not rely too heavily on the respondents' explanations because some of them were inadequate or not clear. However, explanations provided by 358 respondents gave the students' views on atmospheric pressure, and led us to understanding the thought process behind the systematic loss.

In order to understand such processes, we need to identify false positives and the students' thought processes behind them. A false positive is a correct response without understanding the physics concept being tested in the question, where a true positive is that with correct reasoning. This identification-positive responses are either true positives or false positives-is within the framework of classical test theory. However, modern educational research shows that the process of solving physics problems is not simple $[8,9]$. Especially when students are novices, their knowledge has a fragmented nature. The students' thought process in answering a question involves activation of these fragments of knowledge. A pattern that tends to activate together with a high probability is often referred to as a schema. Students activate schemas and organize them to reach their answers. This activation is context dependent and appears as a probabilistic phenomenon. Even if the FCI measures students' commonsense beliefs rather than the connection and organization of physics concepts, as was explained by Hestenes et al. [1], its score is not free from that unstable process [10]. Despite this uncertainty, when a fluctuation appears randomly in the same way for both the pretest and the post-test, the influence on Hake's normalized gain or Cohen's $d$ is negligible [11]. On the other hand, a systematic loss is harmful especially for an evaluation of educational effectiveness in a class of low gain. A nonstatistical change of a student's response is caused by their advancement of knowledge and understanding or successful activation of a corresponding schema. This means that a systematic loss is a prominent phenomenon from which instructors could obtain valuable information.

A systematic loss suggests a situation in which the rate of correct responses decreases as a student's ability progresses. This relationship does not fit the assumption used in item response theory (IRT), a monotonically increasing item response function (IRF). Morris et al. used an item response curve (IRC), a simplistic form of IRF, to investigate the students' response to each item $[12,13]$. IRC visually gives the percentage of students for five answer choices including distractors, as a function of total score. Their analysis showed that some wrong answer choices reflect higher student ability levels than others. They demonstrated a valuable analysis using IRC, and then IRC is used to analyze students' responses to some assessments. Ishimoto and Wittman used the IRC of the
Force and Motion Conceptual Evaluation (FMCE) [14] to compare Japanese and American students' views on force and motion [15]. Xiao, and Bao et al. used the IRC of Lawson's classroom test of scientific reasoning (LCSR) [16] to evaluate its validity $[17,18]$. As for the FCI, Eaton et al. used the IRC of pre-instruction and postinstruction to identify students' prevalent alternative world views [19] and recently, Perez-Lemonche $e t$ al. and Stewart $e t$ al. used an IRC to understand the relationship between Newtonian and non-Newtonian thinking [20]. However, we propose a modification of the IRC for the analysis of a single item. The normal IRC uses a proxy of student's ability calculated from correct responses to all items including the target item, which is commonly the total score. As the result of this inclusion, the mathematical formula produces inevitable correlation between the rate of choices (vertical axis) and the proxy (horizontal axis). Regardless of the quality of an item, a curve of correct response starts at $0 \%$ from the worst ability and ended at $100 \%$ to the best ability. In order to remove this automatic relationship, we propose to exclude the response to the target item from the proxy. This modified IRC (MIRC) represents the correlation between the response to one item and the student's ability given by the other items. In this article, we refer to the total score, the number of correct choices by a student to all FCI items, and the partial score, the number of correct choices to 29 items other than Q.29, by $S_{30}$ and $S_{29}$, respectively.

The rest of this paper is organized as follows. Section II provides an overview of earlier reports, with indications that Q.29 is special among the 30 items in the FCI. Section III describes data taking at the University of Hyogo, Hiroshima University, and a high school in Hiroshima City. Section IV provides results and analyses. This includes the class data that show the systematic loss, the analysis of the students' explanations, and confirmation using the MIRC. In Sec. V we will have some discussions with some additional calculations. We conclude this paper in Sec. VI.

\section{FOREGOING STUDIES ON Q.29}

The English version of Q.29 is given in Appendix A [2]. Students are asked which combination of the three suggested forces are acting on a stationary object. The first force is a downward force of gravity. The second force, "an upward force exerted by the floor" is a normal reaction. The third force, "net downward force exerted by the air" is a contribution of air to gravity [1]. Halloun and Hestenes explained this misconception, "if you release a body in a vacuum, it stays where it is...because gravity does not act in a vacuum. Gravity acts only if there is air... a body moves down because of the air that pushes down on the body." [21] In this paper, the rate of students who choose options (A),(B),(C),(D), and (E) are referred to by $R_{(\mathrm{A})}, R_{(\mathrm{B})}, R_{(\mathrm{C})}$, $R_{(\mathrm{D})}$, and $R_{(E)}$, respectively. Here, $R_{(\mathrm{B})}$ is the rate of the correct answer. 
There has been some factor analysis of 30 items, which reported a small loading of Q.29 to main factors [22-26]; some researchers excluded this item from the analysis. In addition, the module analysis [25] and the modified module analysis [26] showed that Q.29 had a weak connection to the other items. We note that Q.29 is the only FCI item in which the object does not move. Especially for novices, a force to a stationary object and a force to a sliding object on ice (appeared in Q.11) could be a different cue for activating "normal reaction" schema. The words, "force exerted by the air," mean different things to a stationary object and to a moving object.

Many IRT analyses gave Q.29 a middle-ranked difficulty parameter and a low discriminating parameter [13,20,27-34]. Wang and Bao reported the worst discriminating parameter to Q.29 [27]. The analysis by Planinic et al. revealed that Q.29 functions differently in non-Newtonian and predominantly Newtonian populations [28]. They also mentioned that some successful students unexpectedly failed on this item. Eaton et al. proposed a partial credit grading model for the FCI based on the polytomous item response theory (PIRT) [32]. They assigned an unreasonable amount of partial credit to option (D). They suggested that Q.29 be examined in more depth by conducting student interviews or giving free response questionnaires for this question.

In addition to the main results of these studies, some authors added comments that "net downward force exerted by the air" was confusing. Semak et al. wrote their experience of interviewing, "Certain students did claim that question 29 was somewhat confusing given their lack of experience with buoyancy along with the wording of the response mentioned above (a distinction between 'downward' and 'net downward')" [21]. Planinic et al. also wrote, "it seems that the net downward force of the air was confusing for students" [28]. The original concept by Hestenes et al. was the contribution of air pressure to gravity, which is common only among very naive students. Therefore, many other students, who did not have that misconception, struggled to imagine a force that they did not know. Morris et al. pointed out that Q.29 extends beyond the Newtonian thinking dimension that the FCI is designed to measure [13].

There were some other suggestions about the difference between pretest and post-test for Q.29. Traxler et al. examined pretest and post-test results separately. They showed that Q.29 was problematic, which meant poor performance, especially at post-test [31]. According to the module analysis by Wells et al. [35] and Yang et al. [36], only option (A) among the 4 distractors functioned exclusively at pretest. Xiao et al. examined the factor structure using the longitudinal measurement invariance (LMI) analysis [37] and reported that Q.29 was special and its residuals varied between pretest and post-test.

Besides these works, Stewart et al. examined the consistency of student responses to contextually different versions of Q.29 [38]. They replaced the office chair on the floor in Q.29 by 6 different objects, a car in a driveway, a boat in lake, a pack on ice, a student on a lab stool, a balloon in the air, or a marble on a table. The rates of correct answers at the pretest were $42 \%-45 \%$ except for the one using a car $(36 \%)$. These rates at the post-test became higher and were $80 \%-85 \%$ except for the one using a balloon (74\%). This suggested that the balloon was different among the 7 objects for high score students. We speculate that the balloon question may have activated a schema related to buoyancy. McCullough and Meltzer also investigated the dependence of students' responses on context (a diary on a nightstand) and gender before the instruction [39]. The rate of correct responses was higher for the diary, where $R_{(\mathrm{A})}=31 \%$ and $R_{(\mathrm{D})}=28 \%$ for the office chair and $R_{(\mathrm{A})}=21 \%$ and $R_{(\mathrm{D})}=21 \%$ for the diary. It suggested considerable context dependence in prelearners.

The other important information is the Japanese IRC obtained from the results of about 5200 high school and undergraduate school students (it includes 437 responses used in this article) directed by Lang [7]. According to the report by Hasegawa et al. $R_{(\mathrm{B})}=77 \%$ at $S_{30}=18$ and lower $R_{(\mathrm{B})}=68 \%$ at higher $S_{30}=24$. This suggested that the systematic loss of Q.29 is not unusual in Japan.

\section{DATA COLLECTION}

\section{A. Data collection for the IRC}

Test results used to calculate our IRC were obtained at the University of Hyogo, Hiroshima University, and a high school in Hiroshima City from 2013 to 2021. Table I shows the students' affiliations and the number of responses. The number of test results was larger than the number of respondents because the same students took the FCI more than once. The affiliations of some students were not identified because we accepted submissions by students who did not want to write their names. A few students did not take the FCI because the results were not reflected in their grades. Figure 1 shows the distribution of 5017 test scores used for the analysis.

We did not use the following test results. We thought that 14 test results were non-serious responses. Their responses had periodic patterns, such as ABCDEDCBABC or CCCCC and more than two of the choices by one student were not popular options. We also ignored seven other posttest results for which the scores were much worse than the pretest scores. Their individual gains were more than $3 \sigma$ away from the variability of the class members' gains. One hundred and eighty-eight test results had one item with no choice or double choice-36 results contained two items of that kind and 13 results contained three items of that kind. Many results had more than 3 items with no choice. Responses that contained only a few choices were used as records of attendance. 
TABLE I. Affiliations of respondents and number of test results. $\left\langle S_{30}\right\rangle$ is a group average of $S_{30}$.

\begin{tabular}{|c|c|c|c|c|c|}
\hline University & \multicolumn{2}{|c|}{ Department and school } & $\begin{array}{c}\text { Used responses } \\
\text { (respondents) }\end{array}$ & $\left\langle S_{30}\right\rangle$ & $\begin{array}{c}\text { Unused } \\
\text { responses } \\
\end{array}$ \\
\hline \multirow{10}{*}{ University of Hyogo } & \multicolumn{2}{|c|}{ School of Engineering } & $1978(1362)$ & 20.4 & 117 \\
\hline & \multicolumn{2}{|c|}{ Department of Material Science, School of Science } & $584(396)$ & 21.5 & 17 \\
\hline & \multicolumn{2}{|c|}{ Department of Life Science, School of Science } & $213(188)$ & 14.8 & 11 \\
\hline & \multicolumn{2}{|c|}{ School of Human Science and Environment } & $334(248)$ & 13.1 & 21 \\
\hline & \multicolumn{2}{|c|}{ College of Nursing Art and Science } & $68(68)$ & 9.8 & 3 \\
\hline & \multicolumn{2}{|c|}{ School of Economics } & $158(158)$ & 10.2 & 11 \\
\hline & \multicolumn{2}{|c|}{ School of Economics and Management } & $42(42)$ & 14.8 & 9 \\
\hline & \multicolumn{2}{|c|}{ School of Business } & $208(202)$ & 9.8 & 16 \\
\hline & \multicolumn{2}{|c|}{ School of Social Information Science } & $23(23)$ & 20.1 & 2 \\
\hline & \multicolumn{2}{|c|}{ Unconfirmed } & $5(5)$ & 16.2 & 0 \\
\hline \multirow[t]{7}{*}{ Hiroshima University } & \multicolumn{2}{|c|}{ School of Engineering } & $223(223)$ & 22.1 & 3 \\
\hline & \multicolumn{2}{|c|}{ School of Science } & $63(63)$ & 25.0 & 0 \\
\hline & \multicolumn{2}{|c|}{ School of Integrated Arts and Science } & $78(78)$ & 21.9 & 1 \\
\hline & \multicolumn{2}{|c|}{ School of Education } & $111(111)$ & 22.5 & 0 \\
\hline & \multicolumn{2}{|c|}{ School of Informatics and Data Science } & $24(24)$ & 20.5 & 0 \\
\hline & \multicolumn{2}{|c|}{ Other schools } & $6(6)$ & 16.2 & 0 \\
\hline & \multicolumn{2}{|c|}{ Unconfirmed } & $6(6)$ & 23.5 & 0 \\
\hline \multirow[t]{4}{*}{ High school in Hiroshima City } & 1 st test & 222 & \multirow[t]{4}{*}{$(247)$} & 8.7 & 25 \\
\hline & 2nd test & 209 & & 15.4 & 38 \\
\hline & 3rd test & 236 & & 18.3 & 11 \\
\hline & 4 th test & 226 & & 20.6 & 21 \\
\hline \multicolumn{3}{|c|}{ Total numbers of responses and respondents } & $5017(3450)$ & & 306 \\
\hline
\end{tabular}

All of the students took the Japanese version of the 1995 edition using paper sheets. However, in 2020 at the University of Hyogo, the tests were administered during online classes. In order to prevent students from keeping a copy, diagrams and long explanations in the questions were shown on slide pages, turned by an instructor. Students submitted answer choices using polling options, where they read the remaining part of the questions and the options.

It was inevitable that the responses would include various biases. However, we do not think that these biases were large enough to change our main result. Our data include 861 modified test results with an addition of sixth distractors to Q.5 and Q.18. It lowered $S_{30}$ by about 0.5 but

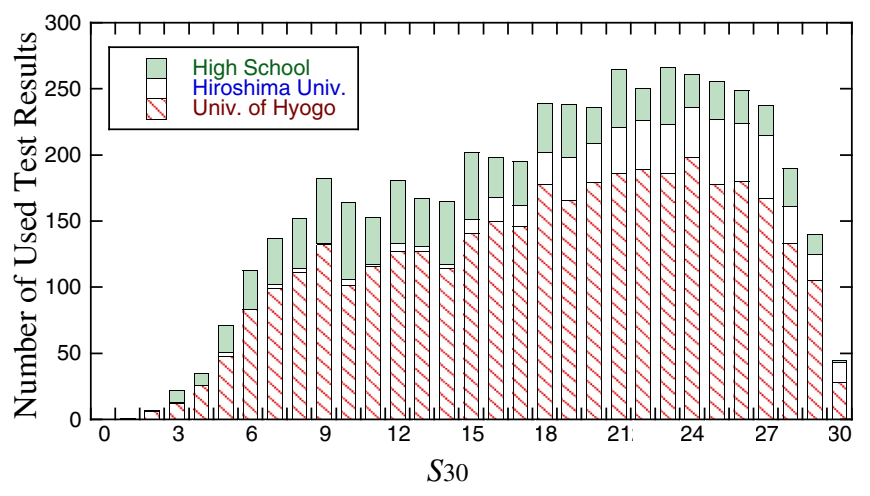

FIG. 1. Distribution of the FCI scores. we ignored this small effect on the total score and focused our analysis and discussions on Q.29. Most of the highschool students took the FCI 4 times, with intervals of about a year. Although there has been no research about the influence of taking the FCI as many as four times, two testretest results by Henderson [40] and Lasry et al. [41] suggested a negligibly small influence from an experience of having taken the FCI in the past. Our data include 94 pairs of test-retest responses in different situations with a one week interval. The class score at the retest was higher because students were required to think logically and were allowed to take enough time [42].

\section{B. Collection of students' explanations}

In 2020 and 2021 at the classes listed in Table II, we asked students to describe what "a net downward force exerted by the air" is using a short answer form (free writing). The request was simple and contained no words, which might be a cue to stimulate special concepts, such as "atmosphere" or "pressure." Three hundred and seventy-six students wrote explanations. Students in classes other than "Basic Concepts of Physics" wrote descriptions just after the pretest. Seventy-one students in Basic Concepts of Physics wrote explanations twice, at 3 weeks after the pretest and one week after the post-test. The explanations after the pretest could be biased because of the long interval of 3 weeks. The explanations changed from the pretest to 
TABLE II. Classes of respondents and number of explanations on Q.29.

\begin{tabular}{|c|c|c|c|c|c|}
\hline \multirow{2}{*}{$\begin{array}{l}\text { University } \\
\text { University of Hyogo }\end{array}$} & \multicolumn{2}{|c|}{ Class } & \multirow{2}{*}{$\frac{R_{(\mathrm{B})}}{83 \%}$} & \multirow{2}{*}{$\frac{\left\langle S_{30}\right\rangle}{21.0}$} & \multirow{2}{*}{$\frac{\text { Explanation }}{102}$} \\
\hline & Basic concepts & Pretest & & & \\
\hline & of physics & Post-test & $84 \%$ & 22.5 & 83 \\
\hline & \multicolumn{2}{|c|}{ Logic (Logical thinking) } & $39 \%$ & 11.8 & 13 \\
\hline & \multicolumn{2}{|c|}{ Scientific reasoning } & $58 \%$ & 18.2 & 24 \\
\hline & \multicolumn{2}{|c|}{ Thermodynamics } & $63 \%$ & 20.1 & 81 \\
\hline \multirow[t]{2}{*}{ Hiroshima University } & \multirow{2}{*}{\multicolumn{2}{|c|}{$\begin{array}{l}\text { Perspectives in physics A } \\
\text { Fundamental physics I }\end{array}$}} & $61 \%$ & 21.3 & 100 \\
\hline & & & $69 \%$ & 22.0 & 26 \\
\hline
\end{tabular}

the post-test because the students learned buoyancy in water during the class.

\section{ANALYSIS AND RESULTS}

\section{A. Systematic loss}

We classify the response pairs for the pretest and the post-test as shown in Table III. $C C$ is the rate of two correct responses in the two tests. $I I$ is the rate of two incorrect responses. $I C$ denotes a gain, where an incorrect response is changed to a correct response. $C I$ denotes a loss, where a correct response is changed to an incorrect response. The net gain is the increase of the total correct responses given by $I C-C I$. In addition, we specify the main distractor (D) of $\mathrm{Q} .29$ and write the change including (D) as $C D, D C$, and $D D$.

Table IV shows the change of students' responses to Q.29 from the pretest to the post-test in three classes in which we observed a systematic loss. In addition to $C C, C I$, $I C$, and $I I$, we show $C D, D C$, and $D D$ in the brackets.

TABLE III. Classifications of response pairs.

\begin{tabular}{lcc}
\hline \hline Class name & Pretest & Post-test \\
\hline$C C$ & Correct (B) & Correct (B) \\
$C I$ & Correct (B) & Incorrect \\
$I C$ & Incorrect & Correct (B) \\
$I I$ & Incorrect & Incorrect \\
$C D$ & Correct (B) & Incorrect (D) \\
$D C$ & Incorrect (D) & Correct (B) \\
$D D$ & Incorrect (D) & Incorrect (D) \\
\hline \hline
\end{tabular}

The increase of the (D) choice was the main reason for the systematic losses. The error intervals in the Table for $C D$ and $D C$ are Bayes confidence interval assuming uniform prior distribution, which exclude $10 \%$ at each upper and lower side. The lower and upper bounds $p_{L}$ and $p_{H}$ were calculated using the following equations:

$$
\begin{aligned}
& \frac{\int_{0}^{p_{L}} p^{n}(1-p)^{N-n} d p}{\int_{0}^{1} p^{n}(1-p)^{N-n} d p}=0.1, \\
& \frac{\int_{p_{H}}^{1} p^{n}(1-p)^{N-n} d p}{\int_{0}^{1} p^{n}(1-p)^{N-n} d p}=0.1,
\end{aligned}
$$

where $N$ is the number of response pairs and $n$ is the number of specific response pairs among them. The integration variable $p$ is the probability of choosing the pair. The positive losses, $C I-I C$, were hardly explained by a statistical fluctuation at least in the classes of Basic Concepts of Physics in 2019 and "Advanced Physics" at a high school.

\section{B. Students' explanations about the third suggested force}

We classified the students' explanations to 7 groups, (I) atmospheric pressure, (II) weight of the air or gravitational force on the air, (III) gravitational force, (IV) air resistance or force by a kind of wind, (V) a paraphrase of "force exerted by the air," (VI) a force which does not exist, and (VII) "I did not understand it" or "I had no idea." Group (I) is then classified into 3 subgroups, (I-1) no more explanation than "atmospheric pressure," (I-2) the sum of

TABLE IV. Change of students' responses to Q.29 from the pretest to the post-test in three classes with systematic loss. The confidence interval was written as the superscript and subscript numbers, respectively. $N$ is the number of students.

\begin{tabular}{lcccccccc}
\hline \hline Physics class & & \multicolumn{3}{c}{$\left\langle S_{30}\right\rangle$} & & \multicolumn{3}{c}{ Rate of response pairs $(\%)$ to Q.29 } \\
\hline Subject names & Year & $N$ & Pre & Post & $C C$ & $C I(C D)$ & $I C(D C)$ & $I I(D D)$ \\
\hline Basic concepts of physics & 2018 & 89 & 20.4 & 22.0 & 61 & $12\left(11_{17}^{8.9}\right)$ & $9\left(6_{10.1}^{3.5}\right)$ & $18(15)$ \\
& 2019 & 53 & 21.0 & 23.1 & 57 & $19\left(13_{22}^{8.8}\right)$ & $0\left(0_{4.2}^{0.2}\right)$ & $24(21)$ \\
Advanced physics (High School) & $2015-2018$ & 214 & 18.6 & 20.6 & 72 & $15\left(10_{13}^{9.1}\right)$ & $6\left(4_{6.5}^{2.9}\right)$ & $7(5)$ \\
\hline \hline
\end{tabular}


TABLE V. Groups of students' explanations and their responses to Q.29.

\begin{tabular}{|c|c|c|c|c|c|c|}
\hline Explanation & Group & (A) & (B) & (C) & (D) & $(\mathrm{E})$ \\
\hline force due to atmospheric pressure & $\mathrm{I}-1$ & 1 & 117 & 2 & 77 & 3 \\
\hline from all directions & $\mathrm{I}-2$ & & & & & \\
\hline result of pressure difference & $\mathrm{I}-2 \mathrm{a}$ & & 11 & & 4 & \\
\hline equal strength & $\mathrm{I}-2 \mathrm{~b}$ & & 7 & & 1 & \\
\hline except area touching the floor & $\mathrm{I}-2 \mathrm{c}$ & & 1 & & 1 & \\
\hline is negligibly small & $\mathrm{I}-3$ & & 9 & & & \\
\hline weight of the air & II-1 & 2 & 25 & & 7 & 1 \\
\hline above the chair & II-2 & & 11 & & 4 & \\
\hline gravitational force & III & 1 & 21 & 2 & 5 & \\
\hline air resistance or force by a kind of wind & IV & 2 & 70 & & 6 & \\
\hline force exerted by air & $\mathrm{V}$ & 2 & 13 & & 2 & \\
\hline a force which does not exist & VI & & 5 & & & \\
\hline did not understand it or have no idea & VII & & 13 & 3 & & \\
\hline no explanation & & 1 & 14 & 1 & 2 & \\
\hline
\end{tabular}

atmospheric pressure from all directions, and (I-3) the force exerted by air is negligibly small. The (I-2) subgroup is classified into 3 sub-sub groups, (I-2a) a resultant force by a difference of pressure, (I-2b) the strength is the same from all direction, (I-2c) the force on all surface except the area touching the floor. Group (II) is classified into 2 subgroups, (II-1) no more explanation than "weight of the air" or "gravitational force on the air," (II-2) with additional expression of "above the chair." Explanations such as "gravitational force on any material or object" were classified into (III). There was no explanation such as "gravitational force on the chair," which meant the contribution of air to gravity. The classification between the 3 groups, (I-1), (II), and (III), was not clear because students with the understanding of atmospheric pressure explained the force using words of "weight of the air" or "gravity." For example, one student classified into (I-1) wrote, "I thought it was gravity. I had an image of a weight of the air above the object. The atmospheric pressure is related to gravity." Students did not always explain the meaning of "net," however, some students of group (IV) wrote that "the resultant force of gravity on the object and air resistance because there is no downward force other than the gravity." Nine students in group (IV) wrote "combined force of air resistance and gravity" and none of them chose (D). One student in group (I-2b) wrote that "net" did not make sense because the combined force is zero. In addition to the 16 responses in group (VII), at least 8 responses were not sure about the meaning and wrote, "It probably means...". Table V shows the number of responses in each group and the choices to Q.29. In this sub-section we focus our discussion on two main choices, correct (B) and incorrect (D).

Table $\mathrm{V}$ shows that most of the (D) choosers belong to group (I-1), which means that they had insufficient understanding about atmospheric pressure. The most probable reason for their incorrect choice was the misconception that "fluid pressure only acts downward" or similar variations.
There were a considerable number of students who were not aware of this misconception and correctly chose (B). Seventy students in group (IV) correctly chose (B) with the reasoning that there is "no resistant force exerted by air because the chair is not moving" or "no downward air flow." Nine students in group (I-3) chose (B) with the reasoning that "force by air is negligibly small." It is possible that some of students in group (I-1), (II), and (III) chose (B) with the same reasoning. One student in group (VII) wrote the reason for choosing (B), that was, "I did not choose that force because I did not know it." These students could change their choice from (B) to (D) by learning more. This is the most possible scenario of the systematic loss that we have observed. Their responses were false positives.

Figure 2 shows the score distribution of responses with different explanations, which is consistent with the above scenario. The false positives, (B) choosers in groups (I-3), (IV), (VI), and (VII) appeared at lower $S_{29}$. On the other hand, both positives and negatives, (B) choosers and (D) choosers, in groups (I-1), (I-2), (II), and (III) appeared at higher $S_{29}$. The ratio of (D) choosers to (B) choosers in

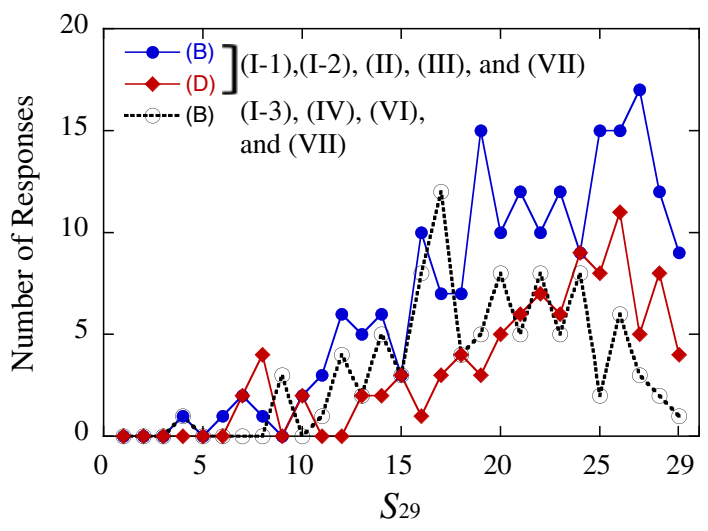

FIG. 2. Score distribution of (B) choosers and (D) choosers with different reasoning. 

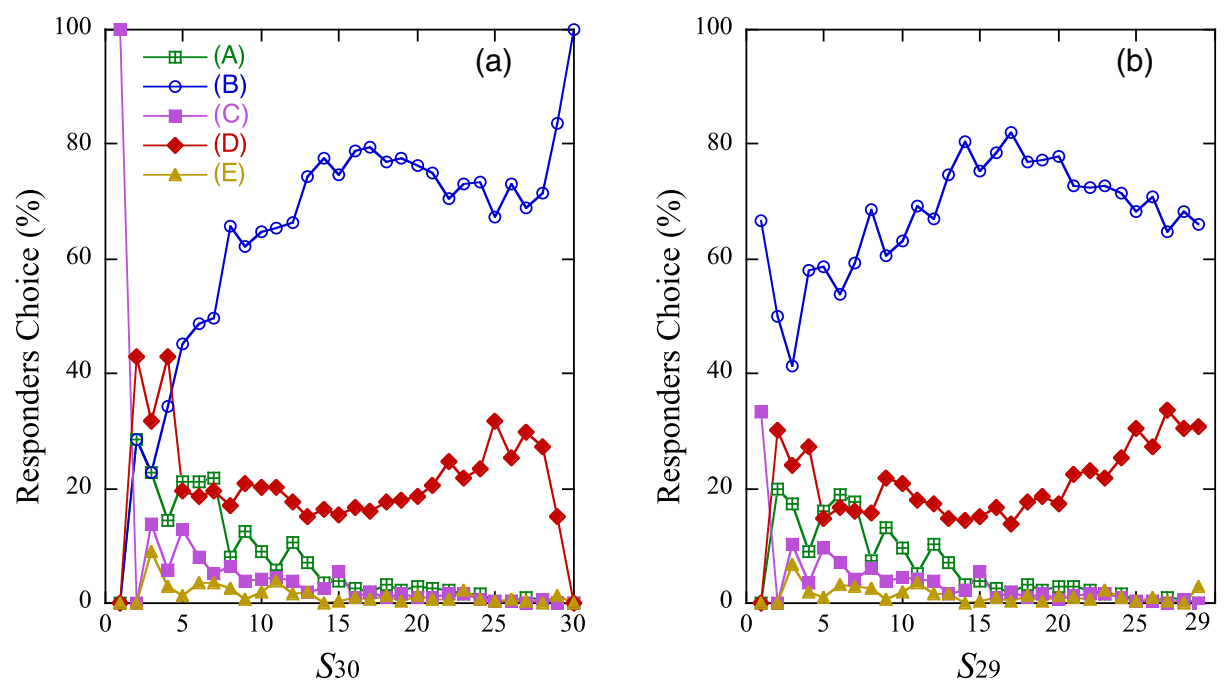

FIG. 3. Item response curve of Q.29. (a) Normal IRC using the score of 30 Items. (b) Modified IRC using the score of 29 items other than that of Q.29 as a proxy of the students' ability.

the latter group was roughly 1 to 2 . We saw no clear dependence of this ratio on $S_{29}$.

The changes of responses of 71 students who took both the pretest and the post-test could be understood in the framework of the above scenario. Although we did not observe many changes, five students changed their choice from (B) to (D). They were in a large composite group consisting of (I-1), (I-2), (II), and (III) at the pretest and at the post-test. Seven students changed their choice from (D) to (B) and 6 of them stayed in the above composite group. One student moved from group (IV) to (I-3). Many students who kept positive responses changed their explanations. Eighteen students in group (IV) and one in group (VI) at the pretest moved to the composite group at the post-test.

\section{Modified item response curve}

Figures 3(a) and 3(b) show the normal IRC and the modified IRC (MIRC) of Q.29. In the normal IRC $R_{(\mathrm{B})}$ rises up from $0 \%$ to $100 \%$ with $S_{30}$. Simply because incorrect response to Q.29 is the necessary condition for $S_{30}=0$ and correct response to Q.29 is the necessary condition for $S_{30}=30$. The rises of $R_{(\mathrm{B})}$ in Fig. 3(a) at $S_{30} \leq 8$ and at $S_{30} \geq 27$ are the results of the calculation formalism. Figure 3(b) is the MIRC, which proxy is $S_{29}$, the score of 29 items other than Q.29. MIRC does not contain that explicit conditioning, then $R_{(\mathrm{B})}$ has a broad peak and $R_{(\mathrm{B})} \approx 50 \%$ at the lower end $\left(S_{29}=0\right)$ and $R_{(\mathrm{B})} \approx 65 \%$ at the higher end $\left(S_{29}=29\right)$.

In Fig. 3(b) $R_{(\mathrm{D})}$ monotonically rises from $S_{29} \approx 15$. More than $30 \%$ of the students who answered all of the other 29 items correctly $\left(S_{29}=29\right)$ chose the incorrect (D). $R_{(\mathrm{D})}$ is still rising at $S_{29}=29$ and looks as if it has a peak at $S_{29}>29$. This suggests that a considerable number of students who correctly chose (B) at the highest $S_{29}=29$ would change their correct choice to incorrectly choose (D). The distractor (D) is most sensitive to high ability beyond the scope of the other 29 items. In discussing the ability of expert students, Hestenes et al. wrote that only two of 16 graduate students understood buoyancy [1]. Their experience was consistent with our result that Q.29 detects buoyant concept, which is beyond the scope of the other 29 items.

On the other hand, at the lowest level, $S_{29}=0$, roughly $50 \%$ of students correctly chose (B). This means that Q.29 has no strong distractor at the lowest level of the scope of the other 29 items.

The above characteristics at both ends become clear with the MIRC, and not clear in the normal IRC. The MIRC visually confirmed comments by many of the researchers presented in Sec. II that Q.29 was beyond the scope of the FCI.

\section{DISCUSSION}

\section{A. Extrapolation of modified IRC}

The moderate slopes at the higher and the lower ends of the MIRC enable images with extrapolation to the outside of the scope. Notice that $S_{29}=29$ does not mean $+\infty$ or a single value on the true ability axis, but covers an area from a certain higher point to $+\infty$. The values for $S_{29}=29$ give values at a point somewhere in this area. Similarly, $S_{29}=0$ does not mean $-\infty$. Therefore, it is reasonable to imagine rates of choices outside the area of plotted ability. Such an idea for understanding ability for students with a full score is common in IRT, and is known as marginal maximum likelihood estimation (MMLE) [43].

Figure 4 is the schematically simplified extrapolation of our MIRC, where the areas represent the percentages of 


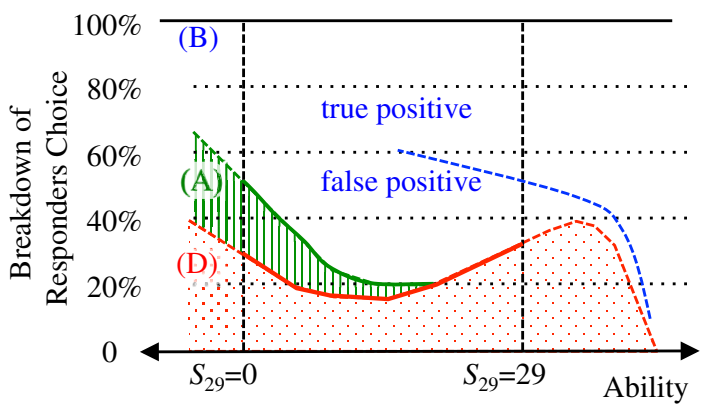

FIG. 4. Schematically simplified image of MIRC. Misconception oriented image of Q.29. The areas (A), (B), and (D) represent the percentage of responses. The rare responses of $(\mathrm{C})$ and $(\mathrm{E})$ are ignored. The broken lines are qualitative estimates.

responses. The horizontal axis is the student's ability, matching $S_{29}$ of our research in the range of $0 \leq S_{29} \leq 29$. The rate of correct response $R_{(\mathrm{B})}$ has a peak at $S_{29} \approx 15$ because students who were not aware of any misconception correctly chose (B). These responses are false positives. On the higher ability side, $d R_{(\mathrm{D})} / d S_{29}>0$ at $S_{29}=29$ although $R_{(\mathrm{D})}$ must be 0 at high enough ability. Then $R_{(\mathrm{D})}$ should be at its maximum at somewhere above $S_{29}=29$. On the lower ability side, $d R_{(\mathrm{A})} / d S_{29}<0$. The $R_{(\mathrm{B})}$ should be smaller than 0.5 at a lower ability than $S_{29}=0$. Figure 4 suggests that Q.29 is sensitive to the ability below the scope of the other 29 items.

\section{B. MIRC difference by country}

In order to see how general our conclusion is, this section compares IRCs reported by different groups. Figure 5 is the MIRCs of options (D), (A), and (B) with those obtained in the U.S. by Morris et al. [13] and Eaton et al. [19]. The MIRCs of the U.S. were transformed from their normal IRCs using equations given in Appendix B.

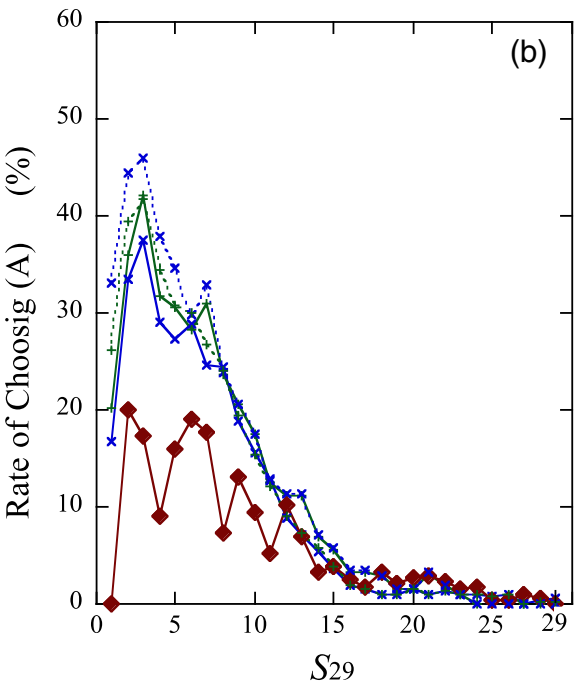

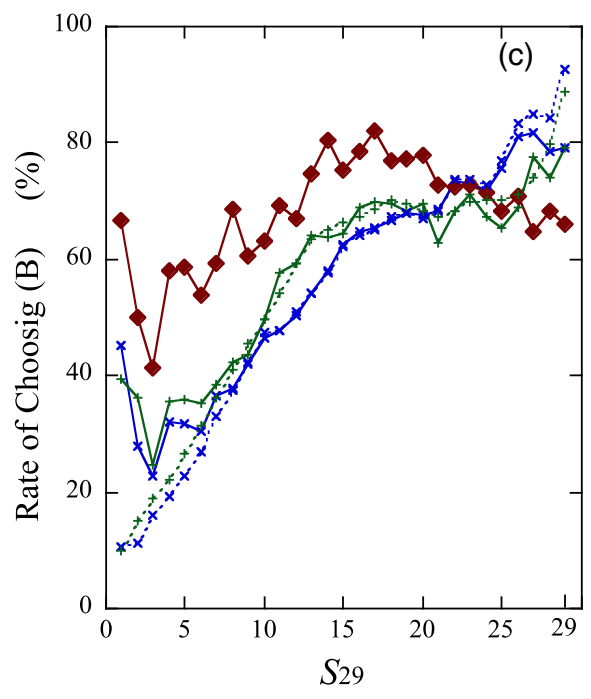

FIG. 5. Comparison of MIRCs obtained in Hyogo and Hiroshima with two MIRCs obtained in the U.S. The MIRCs in the U.S. were transformed from normal IRCs using a score distribution similar to Fig. 1 (solid lines) and the uniform distribution (broken line). 
The transformation depends on a score distribution for students but we did not know it. Then we calculated two MIRCs for each using a uniform distribution and our distribution shown in Fig. 1, with smoothing small bumps in $9 \leq S_{29} \leq 23$. Using the uniform distribution, the unnatural rapid movement appeared in the MIRC by Morris et al. at $27 \leq S_{29} \leq 29$ and it disappeared using our smoothed distribution. The MIRCs in the U.S. are closer to our MIRC using our score distribution rather than those using a uniform distribution.

Figure 5(a) shows the MIRCs of option (D). Its slope at $S_{29}>14$ is positive in our MIRC, roughly zero in the MIRC by Morris et al., and negative in the MIRC by Eaton et al. The MIRC by Morris et al. is much closer to the MIRC by Eaton et al. However, the MIRC by Morris et al. is between the other two MIRCs. The changes of three MIRCs are rather slow compared to those of option (A).

Figure 5(b) shows the MIRCs of option (A). The rates decreased to zero almost linearly, which suggests that this misconception can easily be eliminated. Less Japanese students chose this option probably because they have learned about normal reactions in secondary school. This distractor is not powerful for Japanese high school students and college students.

Figure 5(c) shows the MIRCs of correct option (B). All three curves have break points at $S_{29} \approx 16$. Below and above this point the main distractors are (A) and (D), respectively. Q.29 is more sensitive to the students' ability below this break point. It is possible that the difference between the pre-test and post-test mentioned in Sec. II is due to the difference of the sensitive level of ability of the distractor (A) and (D).

There have been many research projects about the effect of language or culture. However, at least on Q.29, we see no difference of meaning between the Japanese and English versions. It is possible that the difference of the MIRCs between the U.S. and Japan is due to the difference in the physics education systems. Japanese students have learned air pressure and dry friction in secondary school and most college students $(95.3 \%$ of students who wrote explanations) had learned buoyancy in the first year of high school.

\section{Consistency of FCI}

The MIRC explicitly shows the correlation between the response to Q.29 and the other 29 items $\left(S_{29}\right)$ as a function. The bad correlation in Fig. 5(c) suggests better consistency by changing the scoring rule at least in our case. Other than Eaton et al. [32], Morris et al. mentioned a possibility of giving partial credit to a special wrong answer [12]. When Hestenes et al. discussed Q.12 of the 1992 edition, the predecessor of Q.29 of the 1995 edition, they wrote that misunderstanding of buoyancy was acceptable because it is hardly recognized by students at any level [1]. In addition, Planinic et al. [28] indicated that the FCI would probably be a more coherent instrument if Q.29 were excluded from the test, although Q.29 does not degrade measurement.

We calculated the Cronbach's alpha [44] with different scoring rules for the FCI. Cronbach's alpha is a common measure of internal consistency and KR-20 (KuderRichardson formula 20) is a special case of Cronbach's alpha for dichotomous scores [45]. The relationship between Cronbach's alpha and the correlation coefficient is explained in Appendix C.

The KR-20 of the test results used for our IRC was 0.8922 , which was in the range of the KR-20 of the FCI reported in some places (0.8-0.9). When we eliminate Q.29 and make the FCI a 29-item assessment, the KR-20 of the test is improved to 0.8950 . This small increase indicates that the bad correlation between $R_{(\mathrm{B})}$ and $S_{29}$ in Fig. 3(c) works negatively for our group of students. The third choice was to give a half credit to (D). The 30 items with the new scoring rule also improved the KR-20 to 0.8944 (in this case, Cronbach's alpha). This partial credit rule improves internal consistency of the FCI but is not recommended because this improvement is much smaller than the variation of KR-20 obtained in many classes. In addition, Fig. 5(c) suggests better consistency in the U.S. with the inclusion of Q.29.

\section{CONCLUSION}

We evaluated the validity of Q.29 in the Force Concept Inventory. The description of the third suggested force, "a net downward force exerted by the air" allowed at least three interpretations. One interpretation is the misconception of the contribution of air to gravity that is common only among very inexperienced students [1]. The second interpretation is air resistance or airflow, which is not connected to a strong misconception for a stationary object. The third interpretation is the atmospheric pressure. Atmospheric pressure is well understood with the concept of buoyancy, which is hardly recognized by students at any level [1]. Although a perfect understanding of buoyancy is not necessary for the correct response, it is accompanied by a misconception, which makes a student to choose distractor (D). We also found variations in physics knowledge which guide students to false positives. This structure of recognizing the third force sometimes produces a systemic loss of Q.29.

We proposed using a modified IRC, which gave different views for an understanding of the item response, especially the consistency with the other items. The information is not a single coefficient, but a function of $S_{29}$. The normal IRC shows automatic correlations near $S_{30}=0$ and $S_{30}=30$, produced by its mathematical formulas. On the other hand MIRC is free from that conditions and suitable for investigating a special distractor, which works for students with the highest ability or lowest ability. MIRC makes it easier to extrapolate the response to outside of the scope of the other items. 
The FCI is known to be powerful because of its misconception based design, rather than the understanding of physics laws. Therefore, when the item is connected to more than two independent misconceptions, the IRC becomes not as simple. Actually, some IRCs of correct choices of the FCI have a multistep structure because of the FCI's design policy.

We investigated the Japanese students' response behaviors to Q.29 and presented the scenario that explained the observed systematic loss. Although we have not seen any reports showing the systematic loss of Q.29 from other countries, it is natural to think that some students in other countries would have the behavior reported in this article.

\section{APPENDIX A: ENGLISH VERSION OF Q.29}

The following English version of Q.29 is available at PhysPort [2].

An empty office chair is at rest on a floor. Consider the following forces:

1. A downward force of gravity.

2. An upward force exerted by the floor.

3. A net downward force exerted by the air.

Which of the forces is (are) acting on the office chair?

(A) 1 only.

(B) 1 and 2 .

(C) 2 and 3 .

(D) 1,2 , and 3 .

(E) None of the forces. (Since the chair is at rest there are no forces acting upon it.)

\section{APPENDIX B: TRANSFORMATION OF IRC}

The function in a normal IRC, $R_{C}\left(S_{30}\right)$, is transformed into a function in a modified IRC, $R_{M, C}\left(S_{29}\right)$, assuming the population for each score. Here the subscript " $\mathrm{C}$ " denotes the rate of correct responses whereas incorrect choices are denoted by "(I)." When we assume the students' population for the normal IRC, $N(S)$, the students' population for the modified IRC, $N_{M}(S)$, is given by

$N_{M}(S)=N(S+1) R_{C}(S+1)+N(S)\left[1-R_{C}(S)\right]$.

The rate of correct responses and choosing a distractor (I) are given by

$$
\begin{gathered}
R_{M, C}(S)=\frac{N(S+1) R_{C}(S+1)}{N_{M}(S)}, \\
R_{M,(I)}(S)=\frac{N(S) R_{(I)}(S)}{N_{M}(S)} .
\end{gathered}
$$

for every $S=S_{29}$.

In most cases we suppose that both IRC and MIRC do not depend on a respondents' score distribution. However, the results of measurements do depend because they are accumulations of responses from respondents with various abilities. We will give a simple proof for this, an equation for a single measurement point in MIRC. We assume that the probability of correct response to the reference item, $r_{C}$ is a function of unknown ability $\theta$. We also assume that the probability of scoring $S_{29}=k, p_{k}$, and the population density of respondents $N$ are functions of $\theta$. The expected numbers of responses scoring $S_{29}=k$ is $\int p_{k}(\theta) N(\theta) d \theta$ and correct responses to the target item among them is $\int p_{k}(\theta) N(\theta) r_{C}(\theta) d \theta$. Then the observed rate of correct response to the target item for $S_{29}=k, R_{C, k}$, is given by

$$
R_{C, k}=\frac{\int p_{k}(\theta) N(\theta) r_{C}(\theta) d \theta}{\int p_{k}(\theta) N(\theta) d \theta} .
$$

Obviously $R_{C, k}$ depends on $N(\theta)$ unless $r_{C}(\theta)$ is uniform.

This understanding also provides a basis for the validity of the extrapolation made in Fig. 4. The perfect score for a measurement result does not mean that the group of students will always get the same perfect score. Therefore, the ability of students who always get the perfect score is higher than the average of this group.

\section{APPENDIX C: INTERNAL CONSISTENCY}

Cronbach's alpha or coefficient alpha [44] is the most common test score reliability coefficient for single administration. This reliability means internal consistency, whether they are a group of question items that measure the desired characteristics. It is often claimed that a high Cronbach's alpha (e.g., >0.90) indicates a homogeneous test. For a test consisting of $k$ items, the Cronbach's $\alpha$ is given by the following equation:

$$
r=\frac{k}{k-1}\left(1-\frac{\sum_{i=1}^{k} \sigma_{i}^{2}}{\sigma_{X}^{2}}\right) .
$$

Here $\sigma_{i}^{2}$ is a variance of the $i$ th item and $\sigma_{X}^{2}$ is a variance of the total score $X$.

When we add the $(k+1)$-th item and make the $(k+1)$ item test, the Cronbach's $\alpha$ of the exalted test, $r_{+}$is given by

$$
r_{+}=\frac{k+1}{k}\left(1-\frac{\sum_{i=1}^{k} \sigma_{i}^{2}+\sigma_{k+1}^{2}}{\sigma_{X}^{2}+\sigma_{k+1}^{2}+2 \rho \sigma_{X} \sigma_{k+1}}\right) .
$$

Here, $\rho$ is a correlation coefficient between $X$ and $X_{k+1}$. Using this coefficient, the increase of Cronbach's alpha by adding the $(k+1)$-th item is calculated by

$r_{+}-r=\frac{r_{+}}{k^{2}-1}\left(1+k^{2} \frac{\sigma_{k+1}^{2}}{\sigma_{X}^{2}}\right)+2 \frac{k^{2}\left(1-r_{+}\right)+k}{k^{2}-1}\left(\frac{\sigma_{k+1}}{\sigma_{X}}\right) \rho$.

If the $(k+1)$-th item has no correlation with the other items $(\rho=0), r_{+}$is smaller than $r$. A positive correlation $(\rho>0)$ is a necessary condition for $r_{+}>r$. 
[1] D. Hestenes, M. Wells, and G. Swackhamer, Force Concept Inventory, Phys. Teach. 30, 141 (1992).

[2] The latest version of the FCI revised in 1995 and its Japanese version are available at PhysPort, http://modeling .asu.edu/R\&E/Research.html (Retrieved 11/11/2017).

[3] R. R. Hake, Interactive-engagement versus traditional methods: A six-thousand-student survey of mechanics test data for introductory physics courses, Am. J. Phys. 66, 64 (1998).

[4] J. Cohen, Statistical Power Analysis for the Behavioral Sciences (Academic Press, New York, 1977).

[5] K. F. Wilson and D. J. Low, On second thoughts...: Changes of mind as an indication of competing knowledge structures, Am. J. Phys. 83, 802 (2015).

[6] D. J. Low and K. F. Wilson, The role of competing knowledge structures in undermining learning: Newton's second and third laws, Am. J. Phys. 85, 54 (2017).

[7] Y. Hasegawa, S. Kishizawa, T. Yamazaki, J. Yasuda, H. Minoda, M. Goda, T. Murata, and R. Lang, Analysis of mechanics conceptual understanding through "Item Response Patterns", in 2017 Annual meeting of the Physical Society of Japan, 19pC11-5, written in Japanese, https:// www.jstage.jst.go.jp/article/jpsgaiyo/72.1/0/72.1_3378/ _pdf/-char/ja.

[8] E. F. Redish, Teaching Physics with the Physics Suite (John Wiley \& Sons, Hoboken, NJ, 2003), Chap. 2.

[9] A. Perez-Lemonche, J. Stewart, B. Drury, R. Henderson, A. Shvonski, and D. E. Pritchard, Mining students pre-instruction beliefs for improved learning, in Proceedings of the Sixth ACM Conference on Learning, Chicago, USA, June 2019, Article No. 15 (Association for Computing Machinery, 2019), https://doi.org/10.1145/ 3330430.3333637.

[10] D. Hestenes and I. Halloun, Interpreting the force concept inventory: A response to March 1995 critique by Huffman and Heller, Phys. Teach. 33, 502 (1995).

[11] L. Bao, Theoretical comparisons of average normalized gain calculations, Am. J. Phys. 74, 917 (2006).

[12] G. A. Morris, L. Branum-Martin, N. Harshman, S. D. Baker, E. Mazur, S. Dutta, Taha Mzoughi, and V. McCaule, Testing the test: Item response curves and test quality, Am. J. Phys. 74, 449 (2006).

[13] G. A. Morris, N. Harshman, L. Branum-Martin, E. Mazur, T. Mzoughi, and S.D. Baker, An item response curves analysis of the Force Concept Inventory, Am. J. Phys. 80, 825 (2012).

[14] R. K. Thornton and D. R. Sokoloff, Assessing student learning of Newton's laws: The Force and Motion Conceptual Evaluation and the Evaluation of Active Learning Laboratory and Lecture Curricula, Am. J. Phys. 66, 338 (1998).

[15] M. Ishimoto, G. Davenport, and M. C. Wittmann, Use of item response curves of the Force and Motion Conceptual Evaluation to compare Japanese and American students' views on force and motion, Phys. Rev. Phys. Educ. Res. 13, 020135 (2017).

[16] A. E. Lawson, The development and validation of a classroom test of formal reasoning, J. Res. Sci. Teach. 15, 11 (1978).
[17] Y. Xiao, J. Han, K. Koenig, J. Xiong, and L. Bao, Multilevel Rasch modeling of two-tier multiple choice test: A case study using Lawson's classroom test of scientific reasoning, Phys. Rev. Phys. Educ. Res. 14, 020104 (2018).

[18] L. Bao, Y. Xiao, K. Koenig, and J. Han, Validity evaluation of the Lawson classroom test of scientific reasoning, Phys. Rev. Phys. Educ. Res. 14, 020106 (2018).

[19] P. Eaton, K. Vavruska, and S. Willoughby, Exploring the preinstruction and postinstruction non-Newtonian world views as measured by the Force Concept Inventory, Phys. Rev. Phys. Educ. Res. 15, 010123 (2019).

[20] J. Stewart, B. Drury, J. Wells, A. Adair, R. Henderson, Y. $\mathrm{Ma}$, AA. Perez-Lemonche, and D. Pritchard, Examining the relation of correct knowledge and misconceptions using the nominal response model, Phys. Rev. Phys. Educ. Res. 17, 010122 (2021).

[21] I. A. Halloun and D. Hestenes, Common sense concepts about motion, Am. J. Phys. 53, 1056 (1985).

[22] T. F. Scott, D. Schumayer, and A. R. Gray, Exploratory factor analysis of a Force Concept Inventory data set, Phys. Rev. ST Phys. Educ. Res. 8, 020105 (2012).

[23] M. R. Semak, R. D. Dietz, R. H. Pearson, and C. W. Willis, Examining evolving performance on the Force Concept Inventory using factor analysis, Phys. Rev. Phys. Educ. Res. 13, 010103 (2017).

[24] T. F. Scott and D. Schumayer, Conceptual coherence of non-Newtonian worldviews in Force Concept Inventory data, Phys. Rev. Phys. Educ. Res. 13, 010126 (2017).

[25] P. Eaton and S. D. Willoughby, Confirmatory factor analysis applied to the Force Concept Inventory, Phys. Rev. Phys. Educ. Res. 14, 010124 (2018).

[26] J. Stewart, C. Zabriskie, S. DeVore, and G. Stewart, Multidimensional item response theory and the Force Concept Inventory, Phys. Rev. Phys. Educ. Res. 14, 010137 (2018).

[27] J. Wang and L. Bao, Analyzing Force Concept Inventory with Item Response Theory, Am. J. Phys. 78, 1064 (2010).

[28] M. Planinic, L. Ivanjek, and A. Susac, Rasch model based analysis of the Force Concept Inventory, Phys. Rev. ST Phys. Educ. Res. 6, 010103 (2010).

[29] T. F. Scott and D. Schumayer, Students' proficiency scores within multitrait item response theory, Phys. Rev. ST Phys. Educ. Res. 11, 020134 (2015).

[30] E. Brewe, J. Bruun, and I. G. Bearden, Using module analysis for multiple choice responses: A new method applied to Force Concept Inventory data, Phys. Rev. Phys. Educ. Res. 12, 020131 (2016).

[31] A. Traxler, R. Henderson, J. Stewart, G. Stewart, A. Papak, and R. Lindell, Gender Fairness within the Force Concept Inventory, Phys. Rev. Phys. Educ. Res. 14, 010103 (2018).

[32] P. Eaton, K. Johnson, and S. Willoughby, Generating a growth-oriented partial credit grading model for the Force Concept Inventory, Phys. Rev. Phys. Educ. Res. 15, 020151 (2019).

[33] P. Eaton and S. Willoughby, Identifying a preinstruction to postinstruction factor model for the Force Concept Inventory within a multitrait item response theory framework, Phys. Rev. Phys. Educ. Res. 16, 010106 (2020). 
[34] J.-i. Yasuda, N. Mae, M. M. Hull, and M.-a. Taniguchi, Optimizing the length of computerized adaptive testing for the Force Concept Inventory, Phys. Rev. Phys. Educ. Res. 17, 010115 (2021).

[35] J. Wells, R. Henderson, J. Stewart, G. Stewart, J. Yang, and A. Traxler, Exploring the structure of misconceptions in the Force Concept Inventory with modified module analysis, Phys. Rev. Phys. Educ. Res. 15, 020122 (2019).

[36] J. Yang, J. Wells, R. Henderson, E. Christman, G. Stewart, and J. Stewart, Extending modified module analysis to include correct responses: Analysis of the Force Concept Inventory, Phys. Rev. Phys. Educ. Res. 16, 010124 (2020).

[37] Y. Xiao, G. Xu, J. Han, H. Xiao, J. Xiong, and Lei Bao, Assessing the longitudinal measurement invariance of the Force Concept Inventory and the Conceptual Survey of Electricity and Magnetism, Phys. Rev. Phys. Educ. Res. 16, 020103 (2020).

[38] J. Stewart, M. Miller, C. Audo, and G. Stewart, Using cluster analysis to identify patterns in students' responses to contextually different conceptual problems, Phys. Rev. ST Phys. Educ. Res. 8, 020112 (2012).

[39] L. McCullough and D. Meltzer, Differences in male/female response patterns on alternative-format versions of FCI items, in Proceedings of the 2001 Physics Education Research Conference, Rochester, NY(AIP, New York, 2001), pp. 103-106.

[40] C. Henderson, Common concerns about the Force Concept Inventory, Phys. Teach. 40, 542 (2002).

[41] N. Lasry, S. Rosenfield, H. Dedic, A. Dahan, and O. Reshef, The puzzling reliability of the Force Concept Inventory, Am. J. Phys. 79, 909 (2011).

[42] Y. Shoji, Cognitive acceleration program in undergraduate school, in Proceedings of the 2019 8th International Congress on Advanced Applied Informatics (IIAI-AAI), Toyama, Japan, 7-11 July 2019, available in IEEE Xplore (IEEE, 2019), https://ieeexplore.ieee.org/document/ 8992713.

[43] C. S. Wallace and J. M. Bailey, Do concept inventories actually measure anything?, Astron. Educ. Rev. 9, 010116 (2010).

[44] L. J. Cronbach, Coefficient alpha and the internal structure of tests, Psychometrica 16, 297 (1951).

[45] G. F. Kuder and M. W. Richardson, The theory of the estimation of test reliability, Psychometrika 2, 151 (1937). 